\title{
Spontaneous regression of asymptomatic walled-off pancreatic necrosis
}

\author{
Mateusz Jagielski ${ }^{1}$, Marian Smoczyński ${ }^{1}$, Michał Studniarek ${ }^{2}$, Krystian Adrych ${ }^{1}$
}

\author{
${ }^{1}$ Department of Gastroenterology and Hepatology, Medical University of Gdansk, \\ Gdansk, Poland \\ ${ }^{2}$ Department of Radiology, Medical University of Gdansk, Gdansk, Poland
}

Submitted: 24 March 2018

Accepted: 15 April 2018

Arch Med Sci 2019; 15 (5): 1278-1287

DOI: https://doi.org/10.5114/aoms.2018.75606

Copyright @ 2018 Termedia \& Banach

\section{Abstract}

Introduction: Asymptomatic walled-off pancreatic necrosis (WOPN) should be treated conservatively, irrespective of the extent and size of the necrosis. The aim of this study was to evaluate the efficacy and safety of a strategy involving the observation of patients with asymptomatic WOPN over a long period of time.

Material and methods: This study involved the retrospective analysis of 368 patients hospitalized in our department between 2010 and 2016, due to acute pancreatitis and its consequences in the form of pancreatic and peripancreatic fluid collection.

Results: Walled-off pancreatic necrosis was identified in 168/368 (46\%) patients. 124/168 (74\%) patients with WOPN required interventional treatment due to clinical symptoms arising from the presence of the WOPN. Asymptomatic WOPN was identified in 44/168 (26\%) patients. The mean observation time of patients with asymptomatic WOPN was 417.02 days (range: 47-1149 days). Only 1 out of the 44 patients (2\%) failed to complete the follow-up. Complete regression of WOPN occurred in 30/44 (68\%) patients. The presence of symptoms related to WOPN were identified in $13 / 44(30 \%)$ patients during the observation. The most frequent indication for interventional treatment of WOPN was infection of the pancreatic necrosis, which was identified in $6 / 13$ patients (46\%). Altogether, $137 / 168(82 \%)$ patients with WOPN required interventional treatment.

Conclusions: The majority of patients with WOPN required interventional treatment. This study provided evidence to support the view that careful observation of patients with asymptomatic WOPN is an efficient and safe treatment strategy. Long-term observation of such patients showed that most will experience spontaneous regression of asymptomatic WOPN without any other form of interventional treatment.

Key words: walled-off pancreatic necrosis, pancreatic duct disruption, endoscopic retrograde cholangiopancreatography, acute pancreatitis.

\section{Introduction}

As stated in the Atlanta classification from 2012, an acute necrotic collection (ANC) is formed during the first 4 weeks of acute necrotizing pancreatitis (ANP) [1, 2]. After 4 weeks, and during the late phase of ANP, the ANC is referred to as walled-off pancreatic necrosis (WOPN) [1, 2], which is a pancreatic fluid collection (PFC) consisting of a well-defined

\author{
Corresponding author: \\ Mateusz Jagielski MD, PhD \\ Department \\ of Gastroenterology \\ and Hepatology \\ Medical University \\ of Gdansk \\ 17 Smoluchowskiego St \\ 80-214 Gdansk, Poland \\ Phone: +48583493640 \\ Fax: +48 583493650 \\ E-mail: matjagiel@gmail.com
}


wall and a lumen containing liquefied necrosis and fragments of necrotic tissues [1, 2].

Walled-off pancreatic necrosis is observed in approximately $15 \%$ of patients with severe acute pancreatitis [3]. The main indication for interventional treatment of WOPN is clinical suspicion or documented infection of the necrotic collection [4-6]. Furthermore, patients with clinical symptoms arising from the presence of WOPN require interventional treatment [4-6]. Predominantly, such symptoms originate from the pressure the collection places on neighboring structures and organs, thus leading to obstruction of the gastrointestinal tract or mechanical jaundice [4-6].

Asymptomatic WOPN should be treated conservatively, irrespective of its spread and size [49]. Only a limited number of papers available in the current literature have addressed the natural course of asymptomatic WOPN [7, 10-12]. Consequently, there is still a shortage of data related to the long-term consequences and safety of conservative treatment for patients with WOPN who do not show any clinical symptoms.

The aim of this study was to evaluate the efficacy and safety of the careful observation of patients with asymptomatic WOPN over a long period of time and in the absence of interventional therapy.

\section{Material and methods}

The study was approved by the Ethics Committee of our Medical University. All patients gave their informed consent for endoscopic procedures.

This study involved the retrospective analysis of all patients hospitalized in the Department of Gastroenterology and Hepatology of the Medical University of Gdansk between 2010 and 2016, as a result of acute pancreatitis and its consequences in the form of pancreatic and peripancreatic fluid collections. Walled-off pancreatic necrosis was identified based on the criteria detailed in the revised Atlanta classification in 2012 [1, 2]. A significant number of these patients had been previously treated in other clinical centers for acute pancreatitis and were subsequently transferred to our reference medical center in order to treat the consequences of acute pancreatitis [8,9].

The case of each patient with WOPN (medical documentation and imaging examinations) was discussed in detail during interdisciplinary meetings of senior staff in our medical center, including radiologists, gastroenterologists, and surgeons. Decisions related to the choice of treatment were all made during these meetings.

The decision to use interventional treatment for WOPN was made after considering the complete clinical case and results from imaging examinations, generally those of abdominal con- trast-enhanced computed tomography (CECT). Patients with WOPN who did not show any clinical symptoms related to the presence of a necrotic collection were excluded from any interventional treatment.

Prophylactic antibiotic treatment in patients with acute necrotizing pancreatitis or severe acute pancreatitis was not applied in our medical center. Antibiotics are given to patients with acute necrotizing pancreatitis once infected pancreatic necrosis has been diagnosed on the basis of positive microbial culture of necrotic content (direct evidence of infection). Also, antibiotherapy is started in the case of gas bubbles outside the lumen of the gastrointestinal tract visible in computed tomography and suspicion of infection based on maintenance or arising of new symptoms of systemic inflammatory response syndrome after a minimum of 7 days from the onset of disease (indirect evidence of infected WOPN). Antibiotherapy in this group of patients is also used for extrapancreatic infection. Routine fine needle aspiration biopsy in suspicion of infected necrosis is not performed in our medical center.

Empirical antibiotic therapy (mainly tazobactam with piperacillin) was applied in cases of clinical suspicion of infected WOPN. Furthermore, all patients treated interventionally due to pancreatic necrosis had prophylactic antibiotherapy included on the day of the beginning of the treatment. All patients received antibiotics before the procedure (ciprofloxacin or ceftriaxone with metronidazole). Routinely antibiotic treatment was continued for 2 weeks. In the case of interventional treatment, an aspirate from the collection was sent for microbial culture, after which the appropriate culture-directed modification of antibiotics was made.

Asymptomatic WOPN [7] was identified in patients with WOPN who were in good clinical condition after 4 weeks of commencing ANP and who tolerated oral feeding and did not experience or report any symptoms. This group of patients did not include those with infection of the pancreatic collection.

The patients with asymptomatic WOPN were placed under observation, which consisted of additional outpatient care within the gastroenterological clinic. These patients all underwent imaging control examinations of the abdomen, mostly abdominal CECT, after 3, 6, 12, and 24 months of observation or immediately in cases where patients were suspected of having clinical symptoms related to WOPN. None of the patients with asymptomatic WOPN had antibiotherapy or other conservative treatment during the observation period.

Imaging strategies were used to examine the morphology and integrity of the main pancreatic duct (MPD) during the first 6 months of observation. In patients with peripancreatic necrosis, we 
usually used magnetic resonance cholangiopancreatography (MRCP). Endoscopic retrograde cholangiopancreatography (ERCP) was performed for patients with necrosis covering the pancreatic parenchyma (central necrosis or mixed necrosis) and causing a strong suspicion of disruption in the MPD. Patients remained under outpatient care for 24 months of observation. The dates of the next visit to the gastroenterological clinic and the dates of subsequent examinations were provided on an individual basis. Patients were carefully informed about the need to attend the Emergency Department if they experienced alarming symptoms such as jaundice, intensified abdominal pain or fever for any unknown reason. The patients qualified for interventional treatment of WOPN if any symptoms related to WOPN appeared during the period of observation, regardless of the size of the necrotic collection in the imaging examinations.

Disruption of the MPD was defined as a contrast leak outside the MPD during ERCP or MRCP.

Pancreatic parenchymal necrosis alone (central necrosis) - necrosis of pancreatic parenchyma only, without necrosis of peripancreatic tissues.

Peripancreatic necrosis alone (peripheral necrosis) - necrosis of peripancreatic tissues (mainly of fatty tissues) only, without necrosis of pancreatic parenchyma.

Mixed necrosis (both pancreatic and peripancreatic necrosis) - necrosis of pancreatic parenchyma as well as of peripancreatic tissues.

Complete regression of WOPN was defined as the absence of symptoms and complete regression of the collection, or a collection $<10 \mathrm{~mm}$ in size.

\section{Statistical analysis}

All statistical calculations were performed using the data analysis software system StatSoft Inc. (2011) Statistica version 10.0 (licensed for the Medical University of Gdansk). Quantitative variables were characterized by arithmetic means and standard deviation, along with minimal and maximal values (range). Qualitative data are presented as means of numbers and percentage. Raw data were checked for normality using the Shapiro-Wilk test. Multivariate comparisons were performed using a $t$-test/Mann-Whitney test or the Kruskal-Wallis test with Dunn's test of multiple comparisons for quantitative variables, whereas the $\chi^{2}$ test or Fisher's exact test was applied to qualitative data. Two-tailed tests were carried out after setting a significance level of $p \leq 0.05$.

\section{Results}

Three hundred and sixty-eight consecutive patients were hospitalized in our department be- tween 2010 and 2016, as a result of acute pancreatitis and its consequences in the form of pancreatic and peripancreatic fluid collections. The average time of the acute pancreatitis since the onset of abdominal pain in the group of 368 patients was 47.6 (4-402) days, while the average time of hospitalization was 12.2 (1-123) days.

Walled-off pancreatic necrosis was identified in $168 / 368$ (46\%) of these patients. 44/168 (26\%) patients had asymptomatic WOPN diagnosed. The remaining 124/168 (74\%) patients with WOPN required interventional treatment due to clinical symptoms arising from the presence of WOPN.

Clinical suspicion of infected pancreatic necrosis was recorded in 65/168 (39) patients with WOPN. WOPN infection was diagnosed on basis of positive microbial culture in 57/65 (88\%) patients in whom infection was suspected. The most common pathogens cultured in the necrotic contents were Escherichia coli, Enterococcus faecalis and Staphylococcus epidermidis. In 33 patients with WOPN, sepsis with positive blood culture was observed during hospitalization. 65/168 (39\%) patients with WOPN had empirical antibiotic therapy (mainly tazobactam with piperacillin) applied, because of infected pancreatic necrosis diagnosed based mainly on indirect infection evidence. Appropriate culture-directed modifications of antibiotics were made if the cultures of necrotic contents were positive.

Asymptomatic WOPN was identified in 44 patients (35 males, 9 females; mean age: 45.93 \pm 10.52 years, range: $21-69$ years). The mean observation time was $417.02 \pm 316.92$ days (range: 47-1149 days). Follow-up could not be completed for 1 of the 44 patients (2\%) because he died due to myocardial infarction. The etiology of ANP included alcohol (21 patients), cholelithiasis (18 patients), iatrogenic reasons (3 patients), and hyperlipidemia (2 patients). The mean time of acute pancreatitis was 179.89 (55-395) and the average time of hospitalization due to acute pancreatitis was 29.82 (16-59) days. The mean size of the necrotic collection was $100.21 \pm 25.7 \mathrm{~mm}$ (range: 50-176 mm). Peripheral (peripancreatic) necrosis was confirmed in 19 patients, mixed necrosis (pancreatic and peripancreatic) in 21 patients, and central necrosis (pancreatic) in 4 patients.

Complete regression of WOPN (Figure 1) was observed in 30/44 patients (68\%) (22 males, 8 females; mean age $43.43 \pm 11.69$ years, range: $21-$ 69 years). The most common reason for ANP in this group of patients was cholelithiasis (17 patients). Other less common reasons included alcohol (8 patients), iatrogenic reasons (3 patients), and hyperlipidemia (2 patients). The mean size of the WOPN collection was $90.8 \pm 25.6 \mathrm{~mm}$ (range: 50-176 mm). Peripheral necrosis was observed 

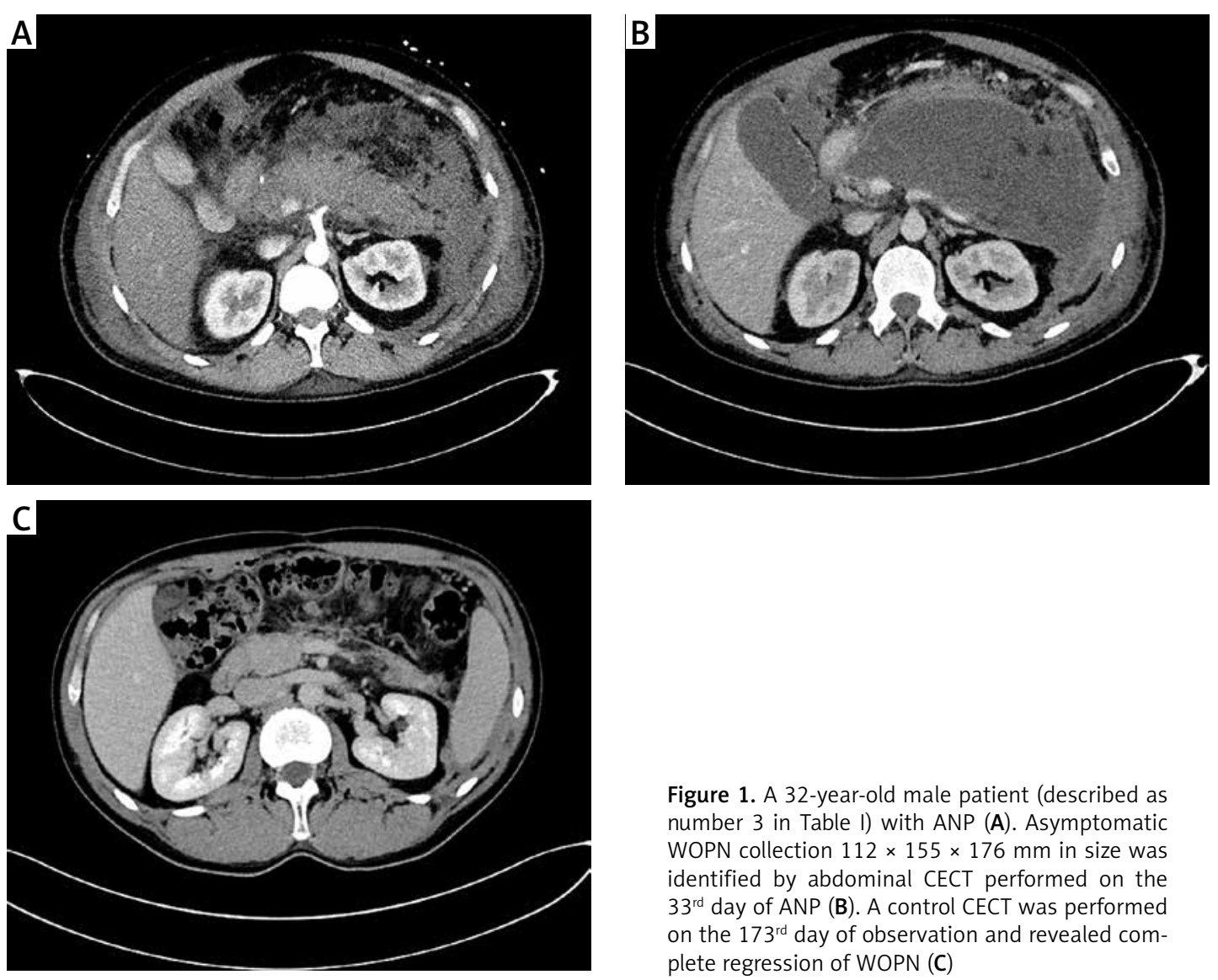

Figure 1. A 32-year-old male patient (described as number 3 in Table I) with ANP (A). Asymptomatic WOPN collection $112 \times 155 \times 176 \mathrm{~mm}$ in size was identified by abdominal CECT performed on the $33^{\text {rd }}$ day of ANP (B). A control CECT was performed on the $173^{\text {rd }}$ day of observation and revealed complete regression of WOPN (C)

in 19 patients, mixed necrosis in 10 patients, and central necrosis in 1 patient. The mean time from the beginning of ANP until the complete regression of WOPN in this group of patients was 188.8 \pm 108.87 days (range: $56-395$ days). The characteristics of patients with complete regression of the necrotic collection are shown in Table I.

The presence of symptoms related to WOPN (Figure 2) during the observation were identified in $13 / 44$ patients (30\%) (12 males, 1 female; mean age: $51.00 \pm 7.44$ years, range: $38-62$ years). The etiology of ANP in 13 patients was related to alcohol. The mean necrotic collection size was 121.9 $\pm 25.9 \mathrm{~mm}$ (range: $80-160 \mathrm{~mm}$ ). Mixed necrosis was identified in 11 patients and central necrosis in 2 patients. MPD disruption during ERCP was identified in 13 patients. The mean time from ANP until the appearance of symptoms was $105.5 \pm 61.26$ days (range: 47-224 days). Indications for starting interventional treatment for WOPN included the suspicion of infection of the necrotic collection $(6 / 13$ patients $(46 \%))$, obstruction of the gastrointestinal tract (5/13 patients $(39 \%))$, and mechanical jaundice (2/13 patients $(15 \%))$. The clinical suspicion of WOPN infection was confirmed in 6 patients on the basis of a positive culture from the necrotic contents. The mean time from ANP until the appearance of symptoms in patients with infected WOPN was $58.33 \pm 12.6$ days (range:

47-73 days). The mean time from the beginning of acute pancreatitis until the appearance of symptoms in the remaining 7 patients who showed the development of signs owing to the pressure created by the necrotic collection (obstruction of the gastrointestinal tract and/or mechanical jaundice) was $145.86 \pm 31.2$ days (range: 64-224 days), which was significantly longer than that in patients with infected WOPN (58.33 vs. 145.86, $p=0.02$ ). All 13 patients in whom the symptoms of WOPN appeared qualified for interventional treatment using minimally invasive techniques. Nine patients were successfully treated endoscopically by transmural drainage. Percutaneous drainage was used in 3 patients with the desired outcome. Only 1 patient required endoscopic drainage combined with percutaneous drainage. Characteristics of the patients with symptoms related to WOPN during the observation period are presented in Table II.

Table III shows a comparison of 30 patients with complete regression of WOPN and $13 \mathrm{pa}-$ tients in whom symptoms originated from the presence of the necrotic collection during the period of observation.

To conclude, between 2010 and 2016 in the Department of Gastroenterology and Hepatology of the Medical University of Gdansk, 137/168 (82\%) patients with WOPN required interventional treatment of pancreatic necrosis. 


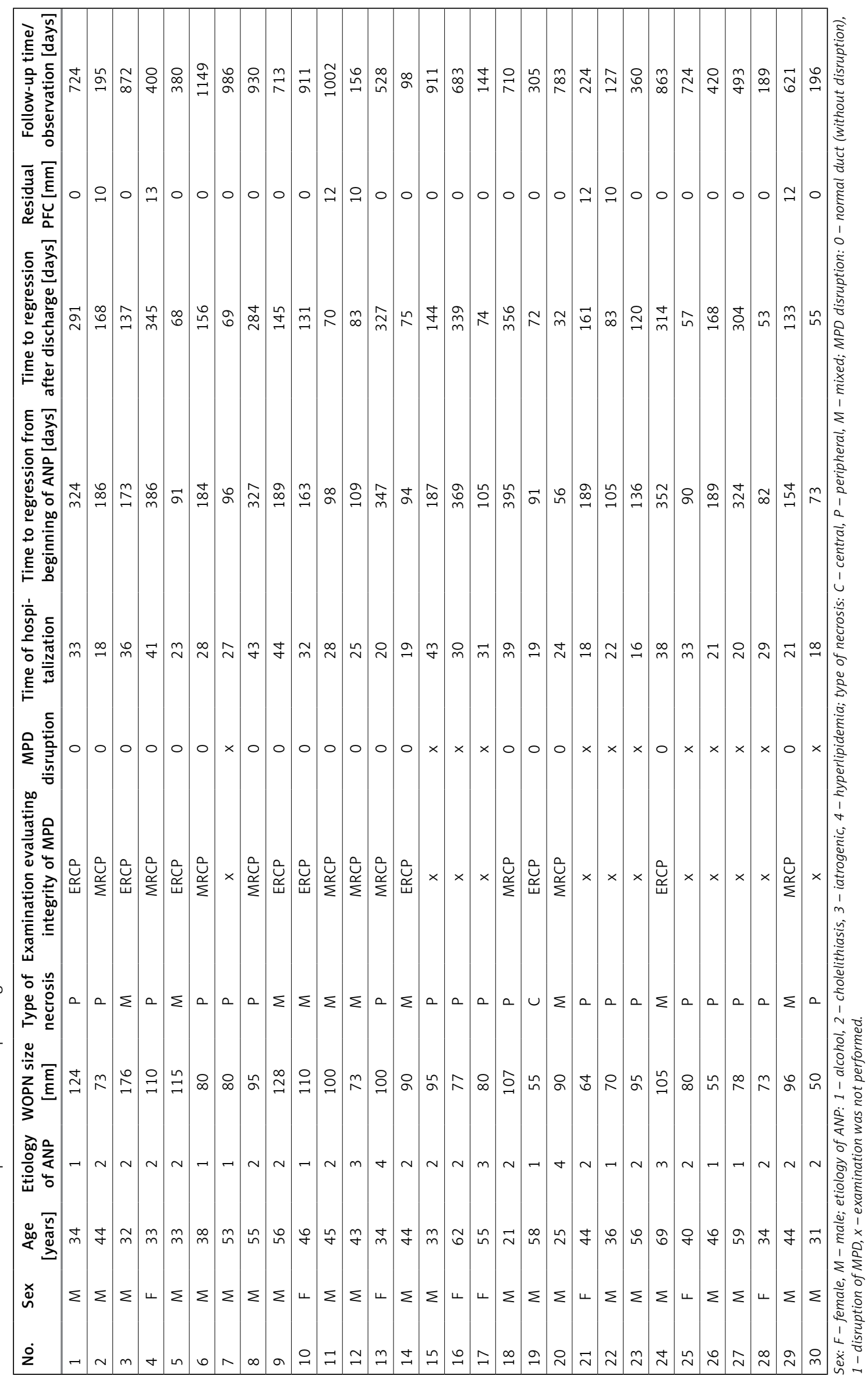



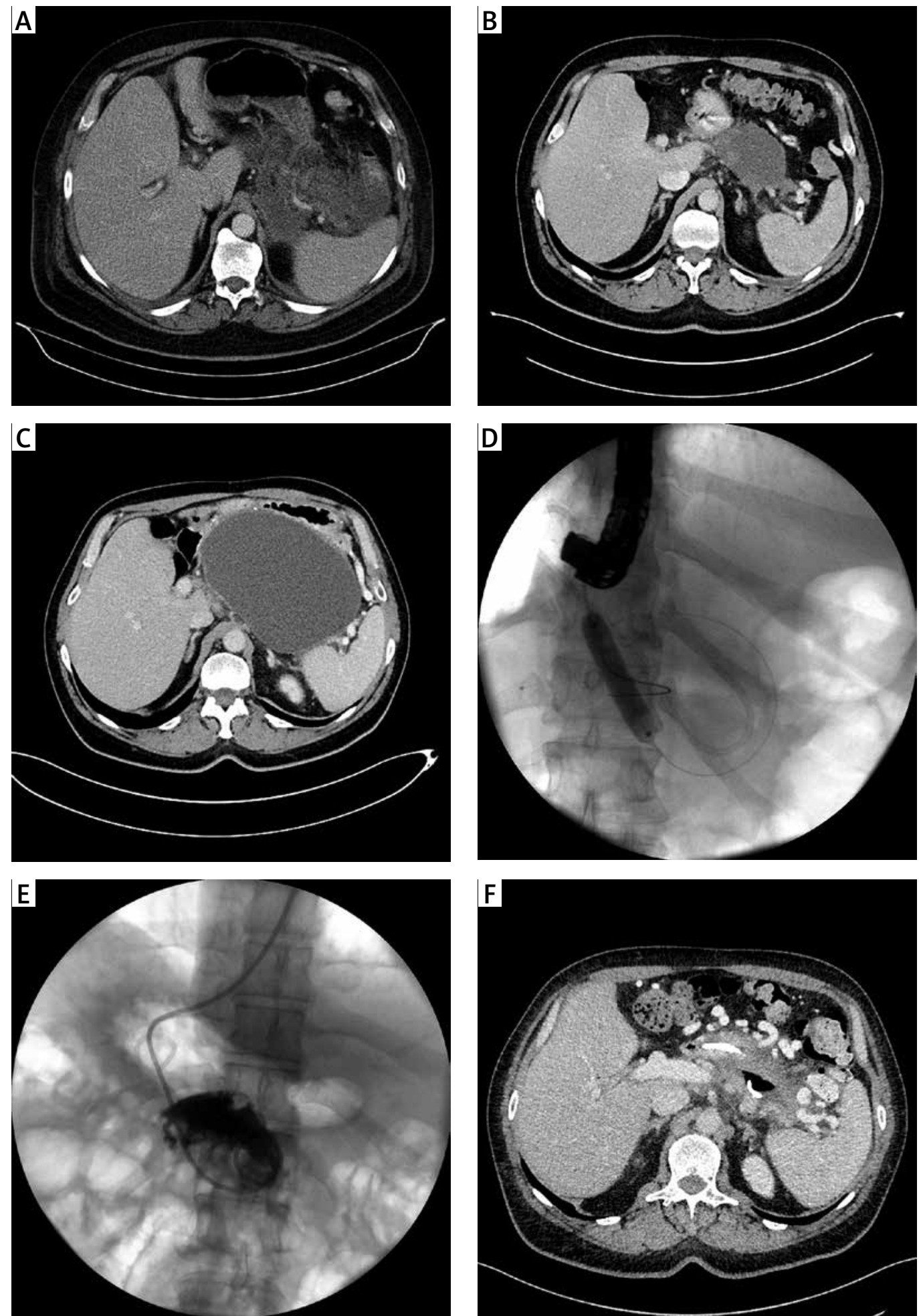

Figure 2. A 53-year-old patient (described as number 5 in Table II) with ANP (A). An asymptomatic collection of WOPN which was $55 \times 95 \times 60 \mathrm{~mm}$ in size (B) was visible in the abdominal CECT performed on the $43^{\text {rd }}$ day of observation. The patient experienced abdominal pain and weight loss caused by obstruction of the gastrointestinal tract by the $163^{\text {rd }}$ day of observation. A WOPN collection $145 \times 220 \times 180 \mathrm{~mm}$ in size, which was pressing upon the lumen of the gastrointestinal tract, was subsequently identified by abdominal CECT (C). The patient was qualified for endoscopic treatment of WOPN (D, E). Endoscopic transmural drainage was performed (D, E). The transmural fistula was widened with a high-pressure balloon to a diameter of $15 \mathrm{~mm}$ during the endoscopic procedure (D). Draining system of WOPN - the stents and the nasal drain led transmurally are noticeable (E). The contrast applied through the nasocystic drain filled the collection of pancreatic necrosis (E). The CECT executed at the end of treatment - complete regression of walled-off pancreatic necrosis is observed (collection less than $3 \mathrm{~cm}$ ) (F). The transmural endoprosthesis is visible in the lumen of the collection $(\mathbf{F})$ 


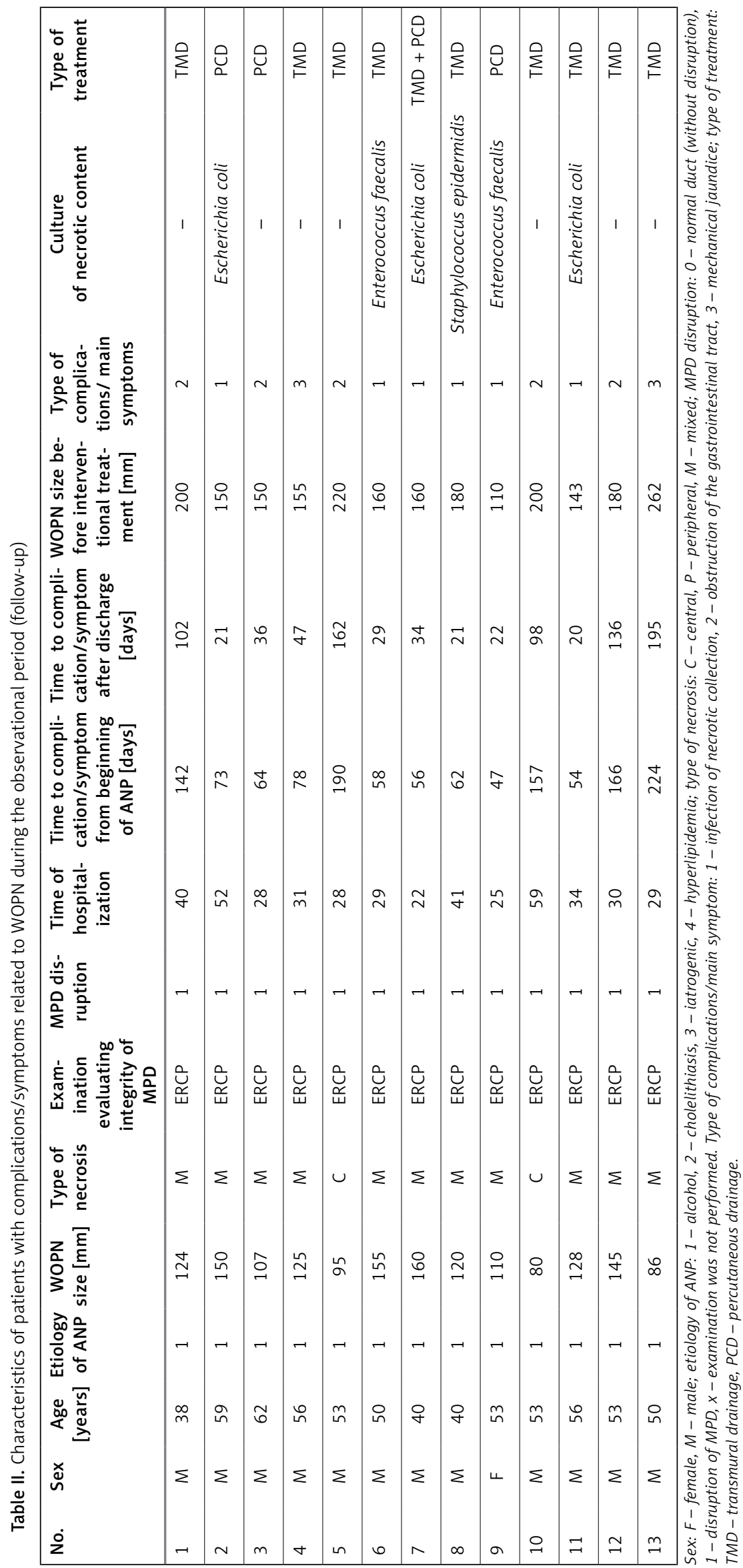


Table III. Characteristics of patients with complete regression of WOPN compared to those of patients in whom symptoms developed due to the presence of a necrotic collection as identified during observation

\begin{tabular}{|c|c|c|c|}
\hline Parameter & $\begin{array}{l}\text { Patients with complete regression } \\
\text { of WOPN }(n=30)\end{array}$ & $\begin{array}{l}\text { Patients with symptoms/ } \\
\text { complications of WOPN }(n=13)\end{array}$ & $P$-value \\
\hline \multicolumn{4}{|l|}{ Sexm \% $(n):$} \\
\hline Female & $26.7(8)$ & $7.7(1)$ & 0.24 \\
\hline Male & $73.3(22)$ & $92.3(12)$ & \\
\hline Mean age (SD) & $43.43(11.69)$ & $51(7.44)$ & 0.02 \\
\hline \multicolumn{4}{|l|}{ Etiology of ANP, \% $(n)$ : } \\
\hline Alcohol & $26.7(8)$ & $100(13)$ & $<0.01$ \\
\hline Cholelithiasis & $56.6(17)$ & 0 & $<0.01$ \\
\hline Iatrogenic & $10(3)$ & 0 & 0.54 \\
\hline Hyperlipidemia & $6.7(2)$ & 0 & $<1$ \\
\hline Mean initial size of WOPN (SD) & $90.8(25.6)$ & $121.9(25.9)$ & $<0.01$ \\
\hline \multicolumn{4}{|l|}{ Type of necrosis, \% $(n)$ : } \\
\hline Central & $3.3(1)$ & $15.4(2)$ & 0.21 \\
\hline Mixed & $33.3(10)$ & $84.6(11)$ & $<0.01$ \\
\hline Peripheral & $63.4(19)$ & 0 & $<0.01$ \\
\hline Disruption of MPD, \% (n) & $0(0 / 18)$ & $100(13 / 13)$ & $<0.01$ \\
\hline
\end{tabular}

\section{Discussion}

Over the last few decades we have encountered significant changes in the treatment strategies used for pancreatic necrosis. These changes have mostly involved a better understanding of the pathogenesis of ANP, improvements in the conservative treatment results of patients with ANP, and the development of minimally invasive treatment techniques for pancreatic necrosis. The natural course of ANP has become more widely understood as a result of a growing body of literature [10-12].

Acute necrotic collection usually develops in the majority (93-100\%) of patients with ANP [10, 11]. Approximately $15-41 \%$ of ANC cases exhibit spontaneous regression, while the rest (49-58\%) develop into WOPN $[10,11]$. Walled-off pancreatic necrosis is a well-defined PFC, which occurs after 4 weeks of ANP and features a collection of liquefied necrosis in its lumen along with fragments of necrotic tissues $[1,2]$. Approximately half of WOPN patients (37-59\%) experience spontaneous regression, without the need for further intervention [10, 11]. The symptoms associated with the presence of WOPN, which represent an indication for the start of interventional treatment, occur in $21-63 \%$ patients $[10,11]$. Nevertheless, the longterm consequences and safety of conservative treatment for patients with asymptomatic WOPN still remain unclear.

The strategy of using conservative observational treatment for patients with asymptomatic WOPN used to be referred to as "watchful wait- ing" [7]. In total, 44 patients with asymptomatic WOPN were subject to long-term observation in the present study. The complete regression of WOPN was documented in $68 \%$ of patients while $30 \%$ of patients showed symptoms associated with the presence of PFC during our observation period and required interventional treatment for WOPN. In 2015, Wroński et al. published the results of an observational study of 16 patients with asymptomatic WOPN [7]; these authors documented the complete regression of WOPN in $44 \%$ of patients [7], while $56 \%$ of patients became symptomatic or developed complications that required interventional treatment [7].

Prospective research, involving a group of 639 patients with ANP, showed that $62 \%$ of patients were successfully treated using a conservative strategy [12], and only $33 \%$ of patients with ANP required interventional treatment for pancreatic necrosis [12]. In our present study, we identified a number of negative predictive factors for spontaneous regression in patients with asymptomatic WOPN: age over 50 years, alcoholic etiology of ANP, necrotic collection over $120 \mathrm{~mm}$ in size, the presence of mixed necrosis, and MPD disruption.

Infection of the pancreatic necrosis can occur in $70 \%$ patients with ANP [13]. Usually this occurs by translocation of intestinal bacterial flora [14]. The most common pathogens involved are Escherichia coli, Klebsiella pneumoniae, Enterococcus faecalis, Staphylococcus aureus, Pseudomonas aeruginosa, Proteus mirabilis, and Streptococcus spp. [13, 15]. A previous study reported that the most common complication of asymptomatic WOPN was an in- 
fection of the pancreatic necrosis, which was discovered in $7 / 16$ patients (44\%) patients, including 7/9 (78\%) patients who had become symptomatic [7]. In our present study, the most frequent indication for the interventional treatment of WOPN was also an infection of the PFC, which was identified in $6 / 44$ patients (14\%), including $6 / 13$ patients (46\%) who required treatment for pancreatic necrosis. Walled-off pancreatic necrosis infection was recognized after a mean of 25 days of observation (range: 20-34 days), in other words, approximately 58 days (range: 47-73 days) after the development of ANP. The most common pathogens cultured from necrotic content in our present study did not differ significantly from those described most recently in the literature [7, 13, 15].

Mixed necrosis (pancreatic and peripancreatic) is quite common during the course of ANP, and is observed in $75-80 \%$ of cases [16]. Van Santvoort et al. reported a much worse prognosis (defined as a larger risk of organ failure and mortality) in a group of patients with central (pancreatic) necrosis in comparison to patients with peripheral (peripancreatic) necrosis [12]. Our present study did not indicate any statistically significant differences in the frequency of central (only pancreatic) necrosis when compared between patients with complete regression of WOPN and patients in whom complications of WOPN occurred and required interventional treatment. However, we did find that peripheral (pancreatic) necrosis occurred more often in patients with complete regression of WOPN during the observational period. On the other hand, mixed (central and peripheral) necrosis was much more commonly observed in patients who required interventional treatment for WOPN complications, despite an initial lack of clinical symptoms related to PFC. The more frequent occurrence of peripheral WOPN in the group of patients with spontaneous regression of the necrotic collection was related to the lack of MPD disruption.

The suspicion of MPD disruption is an indicator for ERCP $[17,18]$. In cases involving confirmation of MPD disruption during ERCP, an endoprosthesis may be inserted into the MPD in order to secure the physiological outflow of pancreatic juice up to the lumen of the duodenum $[17,18]$. It is also recommended to perform secretin-stimulated magnetic resonance cholangiopancreatography (secretin MRCP) to evaluate the MPD when there is no suspicion of MPD disruption and no need to apply endoscopic treatment [19-21]. Secretin MRCP is considered a safe and non-invasive imaging technique, and enables visualization of the whole anatomy of the pancreas, including the pancreatic ducts [19-21]. In our study, ERCP was applied to patients with WOPN when abdominal CECT identified necrosis covering the pancreatic paren- chyma (central necrosis or mixed necrosis), thus generating a suspicion of MPD disruption and the need for endoscopic intervention. In our study, MPD disruption was confirmed in 13 patients with complications of WOPN during the observational period, thus representing $30 \%$ of the study group.

The "step-up approach" is currently an accepted and common strategy for the treatment of WOPN, and consists of initial conservative treatment of symptomatic WOPN with antibiotics and nutritional support [3, 22, 23]. If the symptoms arising from the presence of the necrotic collection remain despite conservative treatment, then interventional treatment is applied using minimally invasive drainage methods (endoscopic, percutaneous or surgical) [3-9, 12, 22-26]. Collectively, the results of our present research study support the "step-up approach". We have shown that the majority of patients with asymptomatic WOPN can be carefully observed, without the necessity for interventional treatment. The efficiency of this approach strategy in our present study was $68 \%$. Interventional treatment, with the application of minimally invasive techniques, should only be used in cases of symptomatic WOPN. All 13 of our patients who had complications originating from WOPN were successfully treated using minimally invasive methods.

Interventional treatment is necessary for patients with clinical symptoms (including infection of necrosis) resulting from the presence of the necrotic collection [3-9]. Combination of several minimally invasive methods of treatment, allowing multiple access to the necrotic collection, is an optimal strategy for treatment of WOPN [3-9, 24-26]. Widening of the access to necrotic areas provides better drainage conditions and increases the efficiency of treatment [3-9, 24-26]. The key to successful treatment of WOPN is the creation of an appropriate irrigation system that enables aggressive active drainage, and providing passive drainage in the later phase [3-9, 24-28]. The method of access to the WOPN should depend not only on the location of the necrosis, but also on the experience of the medical center [3-9, 24-28]. In this retrospective study we found that the majority of patients with WOPN (82\%) required interventional treatment. In the majority of these patients, endotherapy was the only method of treatment. The detailed results of interventional treatment of WOPN in our medical center were presented in our previous publications [8, 9, 24-28].

Our study has some limitations which should be considered when interpreting our findings. First, this study was retrospective in nature and therefore lacked randomization. Second, research was carried out using a select group of patients from a single medical center. Thus, these results cannot be generalized across a larger population. 
In conclusion, the majority of patients with WOPN required interventional treatment due to clinical symptoms arising from the presence of the WOPN. Our study provided evidence that careful observation of patients with asymptomatic WOPN is an efficient and safe treatment strategy. In addition, we described the natural course of asymptomatic WOPN. The long-term observation of patients with asymptomatic WOPN resulted in the majority experiencing spontaneous regression of the necrotic collection. The most frequent complication in asymptomatic WOPN was an infection of the necrotic content, which usually occurred during the first 2 months of observation. The majority of complications of asymptomatic WOPN may be treated using minimally invasive methods of treatment. Nevertheless, further studies are now necessary in order to evaluate the efficacy of this treatment strategy in detail.

\section{Conflict of interest}

The authors declare no conflict of interest.

\section{References}

1. Banks PA, Bollen TL, Dervenis C, et al. Classification of acute pancreatitis-2012: revision of the Atlanta classification and definitions by international consensus. Gut 2013; 62: 102-11.

2. Thoeni RF. The revised Atlanta classification of acute pancreatitis: its importance for the radiologist and its effect on treatment. Radiology 2012; 262: 751-64.

3. da Costa DW, Boerma D, van Santvoort HC, et al. Staged multidisciplinary step-up management for necrotizing pancreatitis. Br J Surg 2014; 101: e65-79.

4. Freeman ML, Werner J, van Santvoort HC, et al. Interventions for necrotizing pancreatitis: summary of a multidisciplinary consensus conference. Pancreas 2012; 41: 1176-94.

5. Gurusamy KS, Belgaumkar AP, Haswell A, Pereira SP, Davidson BR. Interventions for necrotising pancreatitis. Cochrane Database Syst Rev 2016; 4: CD011383.

6. Besselink MG, van Santvoort HC, Freeman ML, et al. Working Group IAP/APA Acute Pancreatitis Guidelines. IAP/APA evidence-based guidelines for the management of acute pancreatitis. Pancreatology 2013; 13 (Suppl 2): e1-15.

7. Wroński M, Cebulski W, Pawłowski W, Krasnodębski IW, Stodkowski M. Walled-off necrosis: safety of watchful waiting. Dig Dis Sci 2015; 60: 1081-6.

8. Jagielski M, Smoczyński M, Jabłońska A, Marek I, Dubowik M, Adrych K. The role of endoscopic ultrasonography in endoscopic debridement of walled-off pancreatic necrosis-a single center experience. Pancreatology 2015; 15: 503-7.

9. Smoczyński M, Marek I, Dubowik M, et al. Endoscopic drainage/debridement of walled-off pancreatic necrosis - single center experience of 112 cases. Pancreatology 2014; 14: 137-42.

10. Sarathi Patra P, Das K, Bhattacharyya A, et al. Natural resolution or intervention for fluid collections in acute severe pancreatitis. Br J Surg 2014; 101: 1721-8.
11. Manrai M, Kochhar R, Gupta V, et al. Outcome of acute pancreatic and peripancreatic collections occurring in patients with acute pancreatitis. Ann Surg 2018; 267: 357-63.

12. van Santvoort HC, Bakker OJ, Bollen TL, et al. Dutch Pancreatitis Study Group. A conservative and minimally invasive approach to necrotizing pancreatitis improves outcome. Gastroenterology 2011; 141: 1254-63.

13. Stamatakos M, Stefanaki C, Kontzoglou K, Stergiopoulos S, Giannopoulos G, Safioleas M. Walled-off pancreatic necrosis. World J Gastroenterol 2010; 16: 1701-12.

14. Seewald S, Groth S, Omar S, et al. Aggressive endoscopic therapy for pancreatic necrosis and pancreatic abscess: a new safe and effective treatment algorithm (videos). Gastrointest Endosc 2005; 62: 92-100.

15. Olson MM, Allen MO. Nosocomial abscess. Results of an eight-year prospective study of 32,284 operations. Arch Surg 1989; 124: 356-61.

16. Sakorafas GH, Tsiotos GG, Sarr MG. Extrapancreatic necrotizing pancreatitis with viable pancreas: a previously under-appreciated entity. J Am Coll Surg 1999; 188: 643-8.

17. Deviere J, Bueso C, Baize M, et al. Complete disruption of the pancreatic duct: endoscopic management. Gastrointest Endosc 1995; 42: 445-51.

18. Varadarajulu S, Noone TC, Tutuian R, Hawes RH, Cotton PB. Predictors of outcome in pancreatic duct disruption managed by endoscopic transpapillary stent placement. Gastrointest Endosc 2005; 61: 568-75.

19. Matos C, Metens T, Devière J, et al. Pancreatic duct: morphologic and functional evaluation with dynamic MR pancreatography after secretin stimulation. Radiology 1997; 203: 435-41.

20. Punwani S, Gillams AR, Lees WR. Non-invasive quantification of pancreatic exocrine function using secretin-stimulated MRCP. Eur Radiol 2003; 13: 273-6.

21. Soto JA, Alvarez O, Múnera F, Yepes NL, Sepulveda ME, Perez JM. Traumatic disruption of the pancreatic duct: diagnosis with MR pancreatography. AJR Am J Roentgenol 2001; 176: 175-8.

22. Nabi Z, Basha J, Reddy DN. Endoscopic management of pancreatic fluid collections-revisited. World J Gastroenterol 2017; 23: 2660-72.

23. van Santvoort HC, Besselink MG, Bakker OJ, et al. A stepup approach or open necrosectomy for necrotizing pancreatitis. N Engl J Med 2010; 362: 1491-502.

24. Jagielski M, Smoczyński M, Adrych K. Endoscopic treatment of walled-off pancreatic necrosis complicated with pancreaticocolonic fistula. Surg Endosc 2018; 32: 1572-80.

25. Smoczyński M, Jagielski M, Jabłońska A, Adrych K Endoscopic necrosectomy under fluoroscopic guidance: a single center experience. Videosurgery 2015; 10: 237-43.

26. Jagielski M, Smoczyński M, Jabłońska A, Adrych K. The development of endoscopic techniques for treatment of walled-off pancreatic necrosis: a single-center experience. Gastroenterol Res Pract 2018, Article ID 8149410; doi:10.1155/2018/8149410.

27. Jagielski M, Smoczyński M, Góreczna-Delich B, Adrych K. Transduodenal drainage of symptomatic walled-off pancreatic necrosis in a patient with ansa pancreatica anatomic variation. Arch Med Sci 2017; 13: 267-9.

28. Smoczyński M, Jagielski M, Siepsiak M, Adrych K. Endoscopic necrosectomy through the major duodenal papilla under fluoroscopy imaging. Arch Med Sci 2018; 14: 470-4. 